\title{
P-AKT PREDICTS
}

PACLITAXEL BENEFIT

Taxanes are effective agents for the treatment of breast cancer. The National Surgical Adjuvant Breast and Bowel Project (NSABP) B28 trial demonstrated a disease-free survival (DFS) benefit for the addition of paclitaxel to anthracycline-based chemotherapy. Currently, no reliable biomarkers exist to predict benefit from taxane-based therapy. Akt has essential roles in cellular processes such as cell growth, proliferation and survival; thus, the $\mathrm{NCl}$ and NSABP trial investigators, lead by Sherry Yang, conducted a study to test whether phosphorylated Akt ( $p$-Akt) could predict a benefit from the sequential addition of paclitaxel to doxorubicin and cyclophosphamide (AC) chemotherapy in women with node-positive breast cancer. "p-Akt at Ser473 is a potential predictive marker for paclitaxel benefit. This is the first large-cohort clinical study that has demonstrated the potential clinical utility of p-Akt-Ser473," explains Yang.

Primary breast tumors from 1,581 patients who were randomly assigned to four cycles of AC or the same regimen followed by four cycles of paciltaxel were assessed. The DFS and overall survival rates were not significantly different among patients with p-Aktnegative tumors. However, in 606 patients with positive p-Akt tumors, the DFS was significantly increased in those receiving paciltaxel (hazard ratio 0.74 , $P=0.02)$. Further analysis revealed that the association between p-Akt-positive tumors and benefit from paclitaxel was evident after stratifying for estrogen receptor and HER2 status.

In summary, p-Akt can predict DFS benefit from the addition of paclitaxel to AC chemotherapy. "Most importantly, once our results are validated, the determination of $\mathrm{p}$-Akt status prior to chemotherapy would allow patients with p-Akt-positive tumors to elect taxanebased chemotherapy, and patients with p-Akt-negative tumors to be spared from such therapy," Yang comments.

Lisa Hutchinson

Original article Yang, S. X. et al. Akt phosphorylation at Ser473 predicts benefit of paclitaxel chemotherapy in node-positive breast cancer. J. Clin. Oncol. 28 ,

2974-2981 (2010) 